\title{
Mental Practice Using Motor Imagery in Dysphagia Rehabilitation: A Survey of Practicing Speech-Language Pathologists
}

\author{
Sarah H. Szynkiewicz, Ph.D., 1 Christina V. Nobriga, Ph.D., ${ }^{2}$ \\ Ashley Cheung, B.S., ${ }^{2}$ Lauren Khoury, B.S., ${ }^{3}$ Morgan Piven, B.S., ${ }^{3}$ and \\ Kassandra Quibin, B.S. ${ }^{2}$
}

ABSTRACT

Mental practice (MP) using motor imagery is recognized as an effective clinical tool in rehabilitative medicine for improving motor performance. Preliminary data using MP in dysphagia rehabilitation are promising, though nothing is known about the current landscape among speech-language pathologists (SLPs) relating to MP implementation. This nationwide study surveys practicing SLPs about knowledge and practice patterns of using MP to gain a better understanding of the current knowledge, as well as perceived benefits and challenges in using MP. Descriptive data are reported and openended questions analyzed for emerging themes using inductive coding. Over half of the participants were familiar or somewhat familiar with motor imagery in the context of dysphagia rehabilitation, though only $16 \%$ of those SLPs reported using MP with a patient. Nearly $75 \%$ of respondents expressed interest in learning more about MP. Emerging themes include factors SLPs perceive to limit patient engagement, evidence-based practice concerns, and therapeutic environmental factors. More research on MP and access to training for clinicians is needed in the area of dysphagia rehabilitation to address acknowledged interest in MP.

KEYWORDS: dysphagia, swallowing disorders, speech-language pathology, mental practice, motor imagery

${ }^{1}$ College of Science and Mathematics, University of South Florida, Sarasota-Manatee, Sarasota, Florida; ${ }^{2}$ Communication Sciences and Disorders, Loma Linda University, Loma Linda, California; ${ }^{3}$ College of Science and Mathematics, University of South Florida, Sarasota-Manatee, Florida.

Address for correspondence: Sarah H. Szynkiewicz, Ph.D., College of Science and Mathematics, University of
South Florida, 350 N. Tamiami Trail, Sarasota, FL 34243 (e-mail: sehegyi@usf.edu).

Semin Speech Lang 2020;41:349-364.

Copyright (C) 2020 by Thieme Medical Publishers, Inc., 333

Seventh Avenue, New York, NY 10001, USA.

Tel: +1(212) 760-0888.

DOI: https://doi.org/10.1055/s-0040-1709204.

ISSN 0734-0478. 
Learning Outcomes: As a result of this activity, the reader will be able to (1) describe the concept of mental practice using motor imagery; (2) summarize previous findings of mental practice and improved motor outcomes; (3) discuss current perceived advantages and limitations of implementing mental practice in dysphagia rehabilitation.

Dysphagia is the medical term for a swallowing impairment. Dysphagia often results from functional or structural abnormalities of the head and neck, and/or esophagus, as well as from damage to the central and peripheral nervous systems. A swallowing impairment can lead to serious medical complications. Furthermore, a swallowing impairment appears to affect more than the person with dysphagia. ${ }^{1,2}$ Stroke, Parkinson's disease, head and neck cancer, and dementia represent some of the more common etiologies of dysphagia.

Management of dysphagia is often behavioral, with a compensatory and/or restorative focus. ${ }^{3}$ Diet modification and other compensatory strategies are used more as immediate strategies to improve the safety and/or efficiency of swallowing during oral intake. However, quality of life and compliance issues often accompany compensatory approaches like diet modification. ${ }^{4}$ Behavioral interventions, such as exercise programs to induce changes in the strength, speed, and timing of musculature important to swallowing, have a restorative focus and some show promise. . $^{5}$ Optimal approaches in restorative approaches like exercise programs have yet to be determined and continued efforts to elucidate the most effective methods are critical. ${ }^{7}$ Drawing from literature in the sports sciences and rehabilitative medicine (occupational and physical therapies) realms may prove beneficial in discovering methods for enhancing the effectiveness of rehabilitative exercises related to swallowing.

One restorative approach currently used and researched within these realms is mental practice (MP) with motor imagery. Motor imagery is the voluntarily driven and self-generated mental representation of a motor task during which there is no overt output. ${ }^{8-11} \mathrm{MP}$ using motor imagery means rehearsing the motor imagery task in a repeated manner or put simply, practicing the motor imagery task. ${ }^{12-15}$ Improved motor performance outcomes have been shown with implementation of MP, particularly alongside physical exercise of the same motor task as compared with physical exercise alone, and MP alone may also be beneficial when physical exercise is not feasible. ${ }^{16-19}$ Rehabilitative medicine intervention approaches like physical practice capitalize on the plastic nature of the cortex in sensorimotor learning. Neural reorganization has been shown with MP in healthy and patient populations, as similar neural areas are activated during motor imagery as during actual motor execution of a task. ${ }^{20}$ From a practice perspective, MP constitutes an attractive therapeutic approach because it does not require physical exertion, it can be performed without direct supervision, and it requires minimal expense and equipment. ${ }^{21}$

Research on clinical implementation of MP is growing quickly in disciplines such as cognitive neuroscience, sport psychology, and other disciplines involving motor learning, in addition to medical and rehabilitation science. ${ }^{14}$ Speechlanguage pathology (SLP) is situated in an exciting position within this interdisciplinary area of interest, as knowledge specific to MP and SLP is limited, but the promising clinical potential is starting to be recognized and explored. $^{21,22}$ For example, investigation of improving lingual strength in typically aging individuals using MP have been initiated, though research in persons with dysphagia is needed. ${ }^{22}$ Researchers are also starting to consider application of MP for motor speech impairment poststroke, highlighting the decades of evidence in other fields to support investigation of MP incorporation in management plans. ${ }^{21}$ Specific to the area of dysphagia rehabilitation, there is a growing body of evidence showing similar neural substrates are shared between motor imagery and motor execution of swallowing and swallowingrelated tasks, as well as supporting the potential benefit in using MP as a clinical tool. ${ }^{22-30}$ For example, Kober and colleagues have shown that swallowing in motor imagery form activates much of the swallowing network throughout 
the entire brain, suggesting that future studies are needed in persons with dysphagia. ${ }^{25}$

Given the promising trends for using MP in dysphagia rehabilitation, we need to better understand the current knowledge and practice, as there is a low presence of this topic in SLP literature, discussion boards, and/or conference sessions. The present study specifically aims to (1) measure the current knowledge and implementation of MP by SLPs in the area of dysphagia treatment, (2) reveal the perception of the utility of MP and its potential effectiveness, and (3) gain a more current understanding of other types of therapy that are most popular for dysphagia treatment. This is critical to identify interest in MP, gaps in knowledge and training, and needs moving forward for research and clinical training with MP in dysphagia.

\section{METHODS}

\section{Recruitment}

Institutional Review Board approval was obtained from both principal investigators (S.S. and C.N.) universities. A convenience sample of SLP participants was recruited via email, alumni social media groups, and American SpeechLanguage-Hearing Association (ASHA) Special Interest Group 13 (SIG-13). A message containing the URL link for the survey was posted to the social media groups and ASHA SIG-13 Community Forum. SIG-13 and social media groups were selected based on their focus on medical settings and swallowing disorders. The URL link took SLPs to the consent form. The informed consent process was presented and granted electronically prior to initiating the survey. Participation in the survey was voluntary and responses were anonymous to the investigators. All potential participants were privy to the participating universities' names and principal investigator's names/credentials. It is unknown if prior relationships existed between the principal investigators and participants, as respondents were anonymous to the investigators.

The inclusion criteria for this study were that participants have at least a master's degree in the field of SLP and be a fully licensed, practicing SLP. Individuals from countries outside of the United States were permitted to participate if they had the equivalent of a license for their country. Participants were also required to work with patients with dysphagia for an average of 1 hour per day. SLP assistants were excluded from this study as they are not involved in developing intervention plans.

\section{Survey}

A survey was developed and disseminated using an online survey platform (Qualtrics, Provo, UT). Questions for the survey were written based on current MP with motor imagery literature review and the principal investigators' clinical experiences in medical SLP. The questions in the survey were created in collaboration between the research teams at each university. The survey was then reviewed by a university department with expertise in survey development. Modification of the survey involved development of unique branches of the survey dependent on a participant's current use of MP in dysphagia management. To ensure a brief yet thorough survey experience, with the hope of increasing response rate, the survey was developed so that it could be completed in 15 minutes or less.

Prior to voluntarily agreeing to participate in the survey study, participants were provided a brief summary to review. The summary first defined motor imagery and MP, then briefly discussed how MP has been implemented in other disciplines (i.e., physical and occupational therapies). Specific examples were provided to demonstrate what type of motor skills may be visually and kinesthetically imagined using MP. The summary ended with a description of how MP may be applicable to the field of SLP. See Appendix A to review the complete survey.

The survey began with a core of similar questions for all participants concerning current level of knowledge of motor imagery. All participants were also asked what intervention techniques they use with persons with dysphagia and if they may be willing to incorporate MP into their patients' management plans. The survey then branched into two sections with questions for those who use MP with their patients and those who do not. Those who use MP in therapy were asked questions regarding therapy tasks, determining patient candidacy, scheduling, monitoring patient progress, perceived advantages of 
MP, and perceived limitations of MP. Those who do not use MP in therapy were asked questions regarding the extent to which they think MP could potentially benefit their patients and what factors may affect their decision to implement this approach. All participants were asked demographic questions at the end of the survey. Participants were allowed to voluntarily skip any question they did not want to answer.

\section{Data Collection}

Data were anonymously collected and stored either in Qualtrics or separately on a passwordprotected laptop. The survey accepted responses for 30 days to allow interested SLPs to participate if willing. No identifying information or protected health information was collected and no physical records were collected. The electronic consent form was presented on the first page of the Qualtrics survey.

\section{Data Analysis}

Descriptive data were used to report single- or multiple-option questions. Open-ended questions were analyzed using inductive coding. Coding was initially performed by two student investigators, with triangulation of coding performed by the two senior principal investigators. Codes for each open-ended question were derived from participant responses-using their own words. Codes were then categorized into emerging themes to support greater understanding of SLPs' perceived benefits and challenges regarding MP.

\section{RESULTS}

\section{Demographic Survey Questions}

All respondents were asked final demographic questions to better understand the context of those responding to the survey. A total of 99 responses were collected for the survey, yet 151 settings were reported, indicating SLPs working in multiple settings. Settings included inpatient acute care $(n=49,32 \%)$, inpatient rehab $(n=$ $32,21 \%)$, outpatient $(n=25,17 \%)$, skilled nursing $(n=25,17 \%)$, private practice $(n=6$, $4 \%)$, and school $(n=2,1 \%)$. Twelve (8\%) reported "Other" settings and 11 were listed. These included long-term acute/long-term hospital $(n=3)$, geriatric home health $(n=3)$, assisted living facility $(n=1)$, early intervention home health $(n=1)$, community transitional care $(n=1)$, university clinic $(n=1)$, and outpatient pediatrics $(n=1)$.

Fig. 1 displays the types of populations the SLPs reported working with most often. Those listed under "Other" $(n=15)$ included head and neck cancer $(n=8)$, spinal cord injury $(n=3)$, trauma/surgery $(n=2)$, trach/vent $(n=1)$, hip fracture $(n=1)$, transplant $(n=1)$, oncology $(n=1)$, critically ill $(n=1)$, postintubation $(n=1)$, debility $(n=1)$, head and neck injuries $(n=1)$, and voice $(n=1)$. The majority of SLPs spend 2 to 4 hours per day with clients with dysphagia $(n=42,49 \%)$, followed by 5 to 7 hours $(n=28,33 \%)$, greater than or equal to 8 hours ( $n=11,13 \%)$, and less than or equal to 1 hour $(n=5,6 \%)(n=86)$. Most respondents reported co-treating: with occupational therapists $(n=$ $51)$, with physical therapists $(n=46)$, with physicians $(n=25)$, with psychologists $(n=7)$, with behavioral therapists $(n=3)$, and with teachers $(n=2)$. Three other professionals were listed for co-treating: CNAs, neuropsychologists, and dietitians. Three SLPs stated "only occasionally," they do not "co-treat, but collaborate with physicians and other therapies," and one stated early intervention co-treating is not reimbursable in his/her state. There were no reports of co-treating with audiologists. Ten (12\%) of the respondents were board-certified specialist-swallowing (BCS-S; $n=85$ ). Over one-third of the SLPs $(n=34,40 \%)$ had 11 or more years of experience, followed by 4 to 6 years $(n=22,26 \%)$, and equal amounts for 7 to 10 and 0 to 3 years $(n=15,17 \%$ each) $(n=86)$. Sixty-four (74\%) SLPs were interested in learning more about MP, 18 (21\%) were "not sure," and $4(5 \%)$ reported no interest in learning more $(n=86)$.

Eighty-six SLPs reported the state in which they were practicing, which the investigators divided into regions of the USA: South $(n=$ $28,33 \%)$, Midwest $(n=23,27 \%)$, West $(n=19$, $22 \%)$, and Northeast $(n=16,19 \%)$. There were 17 states which were not represented; these were primarily in the Pacific, Mountain West, West North Central Midwest, and South Atlantic regions. The investigators were contacted by at 


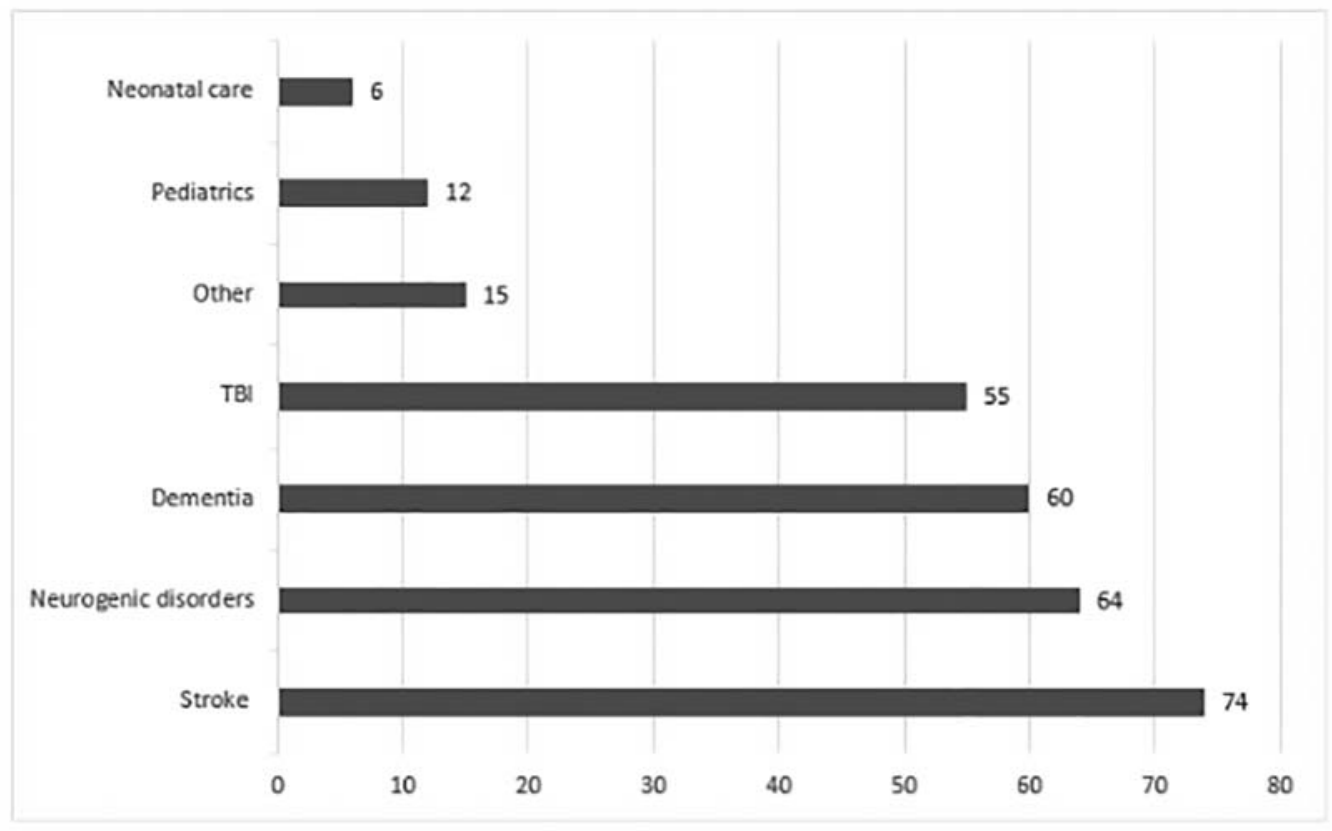

Figure 1 Most common patient populations worked with speech-language pathologist participants. TBI, traumatic brain injury.

least one potential respondent from Europe who was told he or she could participate in the survey as long as he or she held the equivalent of licensure in his or her country. It is unknown whether this person participated. The vast majority of respondents were female $(n=80,93 \%)$, with $5(6 \%)$ males responding, and 1 (1\%) individual who preferred not to answer $(n=86)$. Caucasian was the predominant race/ethnicity $(n=73,85 \%)$, followed by those who preferred not to answer $(n=5,6 \%)$, Hispanic $(n=3,4 \%)$, Asian $(n=2$, $2 \%)$, and African American ( $n=1,1 \%)$. Two (2\%) respondents reported "Other," with one identifying as mixed race/ethnicity. No respondents selected "American Indian or Alaska $\mathrm{Na}^{-}$ tive" or "Native Hawaiian or Pacific Islander" $(n=86)$. Over half of the SLPs were between 2029 and 30-39 years of age, with 23 (27\%) and 29 (34\%) in each group, respectively. Sixteen (19\%) were 40 to 49 and there were $9(10 \%)$ each in the 50 to 59 and 60 or older groups $(n=86)$.

\section{Core Questions for All Participants}

Twenty-two respondents (22\%) were familiar with MP with motor imagery exercise, while 47 (48\%) were not and 30 (30\%) reported being somewhat familiar $(n=99)$. The number of participants refusing to volunteer is unknown. Seven (7\%) reported knowing other SLP colleagues who use MP and 19 (19\%) reported knowing professionals from other disciplines who use it $(n=99)$. Over half of the participants $(n=54$, $55 \%$ ) believed their patients would be willing to incorporate MP with their dysphagia management plan, with 37 (37\%) being unsure, and 8 $(8 \%)$ responding "No" $(n=99)$. Fig. 2 displays the most common techniques that the SLP participants indicated as used for dysphagia rehabilitation. Of the six "Other" responses, ice chips, chin tuck against resistance $(n=2)$, highresolution manometry, shaping and behavioral reinforcement, and modified Frazier free water protocol were given. Sixteen (16\%) SLPs reported having used MP with a patient before and 83 $(84 \%)$ reported not having used it $(n=99)$.

\section{Respondents Who Have Not Used Mental Practice}

Respondents who do not use MP were asked their clinical opinion for potential applicability, use as a supplemental approach, and anticipated patient response if they were to use $\operatorname{MP}(n=83)$. 


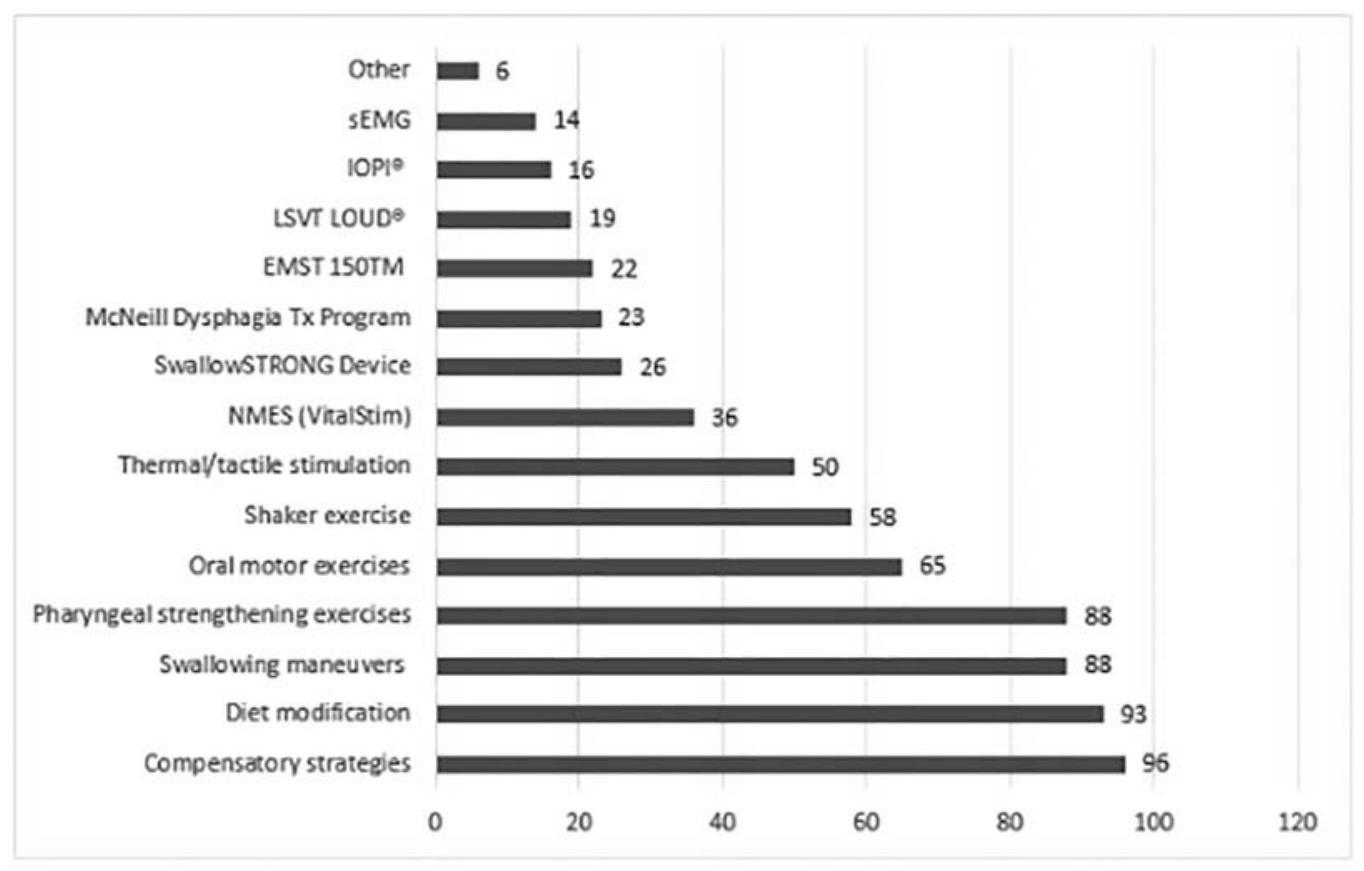

Figure 2 Most common dysphagia treatment techniques reported.

Understanding of MP was based on the descriptive information provided at the beginning of the survey (see Appendix A) and any previous understanding they had acquired prior to taking the survey. The majority of responses to these clinical opinion questions were a "small" to "moderate" degree. Fig. 3 displays these results. Factors affecting the likelihood to implement or try MP included (participants could select all that apply) more evidence in the literature $(n=$ $63,76 \%)$, self-efficacy for patient $(n=53,64 \%)$, ease of implementation $(n=39,47 \%)$, perception by others in their field $(n=16,19 \%)$, and "Other" $(n=8,10 \%)$. "Other" responses primarily centered on the ability of the patient to participate (e.g., acutely ill, cognitive limitations, age/pediatrics), with limited time for treatment being listed by one respondent.

SLPs were asked in an open-ended question to provide any perceived challenges that would deter them from implementing MP. Sixty-nine respondents addressed this question, with a total of 124 responses given. Their responses were inductively coded. The most common perceived challenge that emerged was cognitive level of patient/multiple handicaps $(n=35)$, followed by patient motivation/par- ticipation/attitude $(n=17)$, lack of clinician knowledge/training $(n=12)$, lack of research $(n=11)$, determining patient imaging ability $(n=4)$, pediatrics/client's age $(n=3)$, insufficient therapy time $(n=2)$, and distracting treatment environment $(n=1)$. One respondent was unsure, while another said there were no perceived challenges that would deter them from implementation (see Fig. 4).

\section{Respondents Who Have Used Mental Practice}

Four SLPs using MP distinguish between external and internal motor imagery $(n=9)$. Respondents reported using MP with a variety of traditional dysphagia therapeutic approaches (see Fig. 5), with "Other" described as voice therapy. Seven SLPs alternate between mental and physical exercise repetitions or sets within a session, three divide the session into two parts (mental and physical exercise), two encourage only mental exercise outside of therapy sessions, and one reported alternating days of mental and physical exercise. One specified providing initial training and reiteration education as needed. The typical schedule for patients using MP 


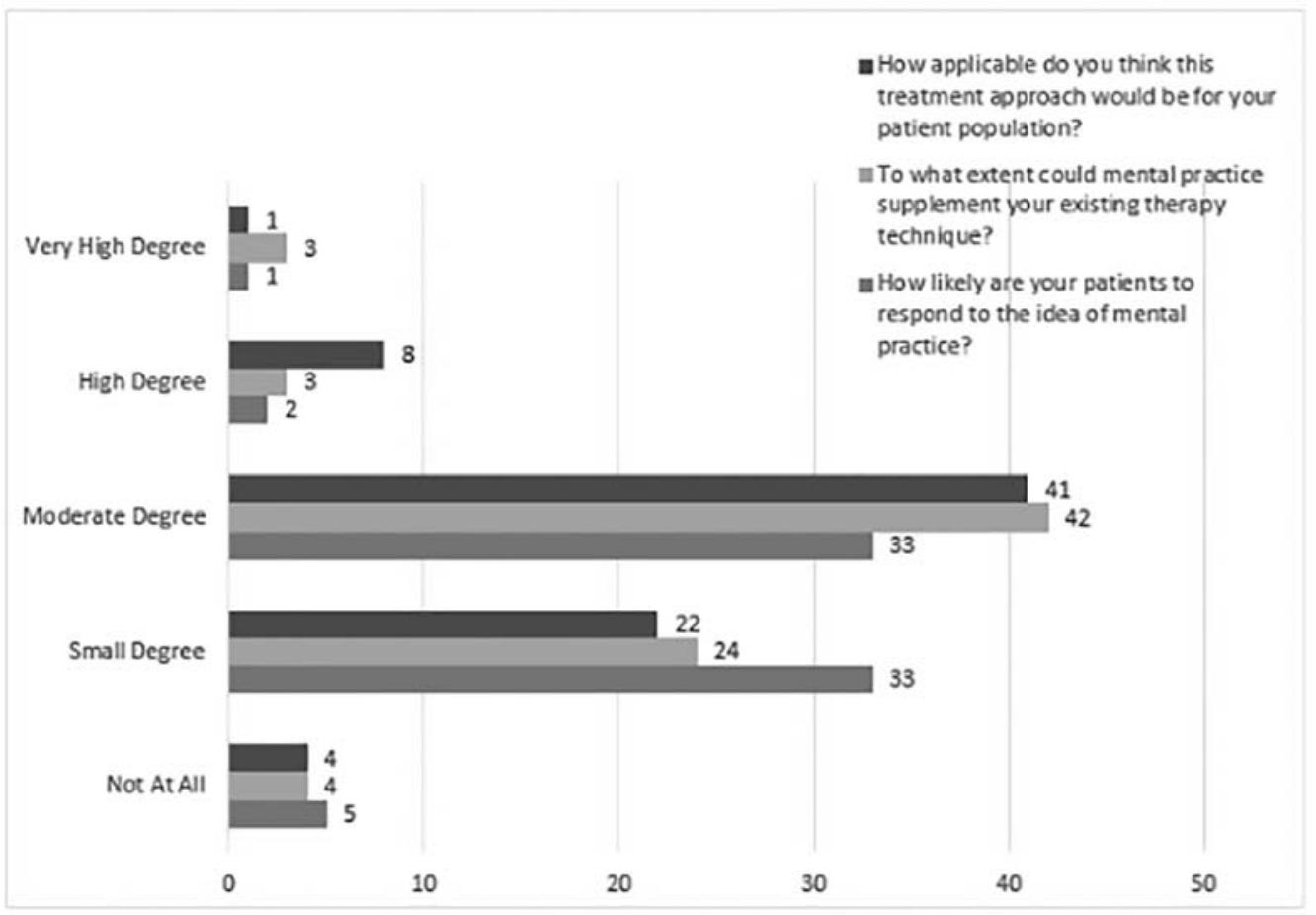

Figure 3 Clinical opinion for usefulness, applicability, and patient responsiveness to mental practice.

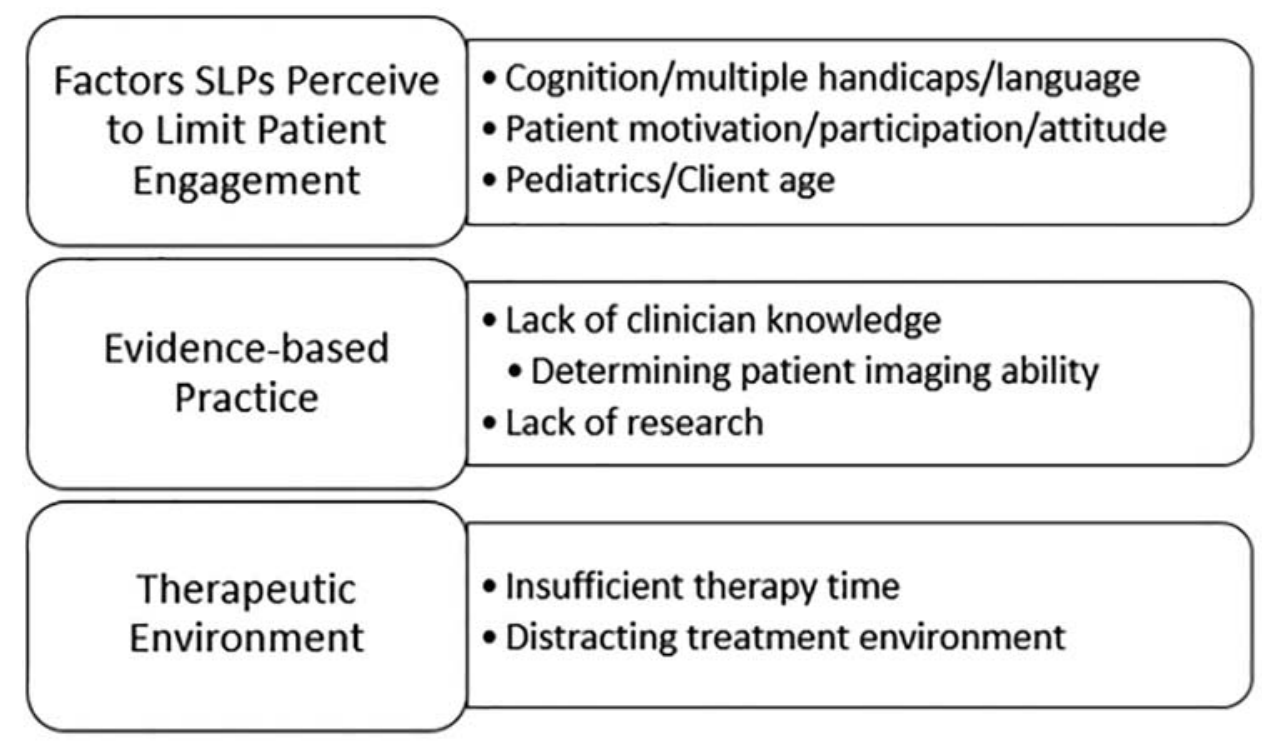

Figure 4 Codes organized by themes for factors deterring mental practice implementation.

was reported by three SLPs to be three to five times per week, three reported several times per day, two reported twice per day, with another two selecting daily, and one indicating less than three times per week $(n=11)$. Fig. 6 displays how respondents monitor patient progress and performance outcomes, with the "Others" being reported as Expiratory Muscle Strength Trainer/Penetration-Aspiration Scale changes and not monitoring effects of MP. 


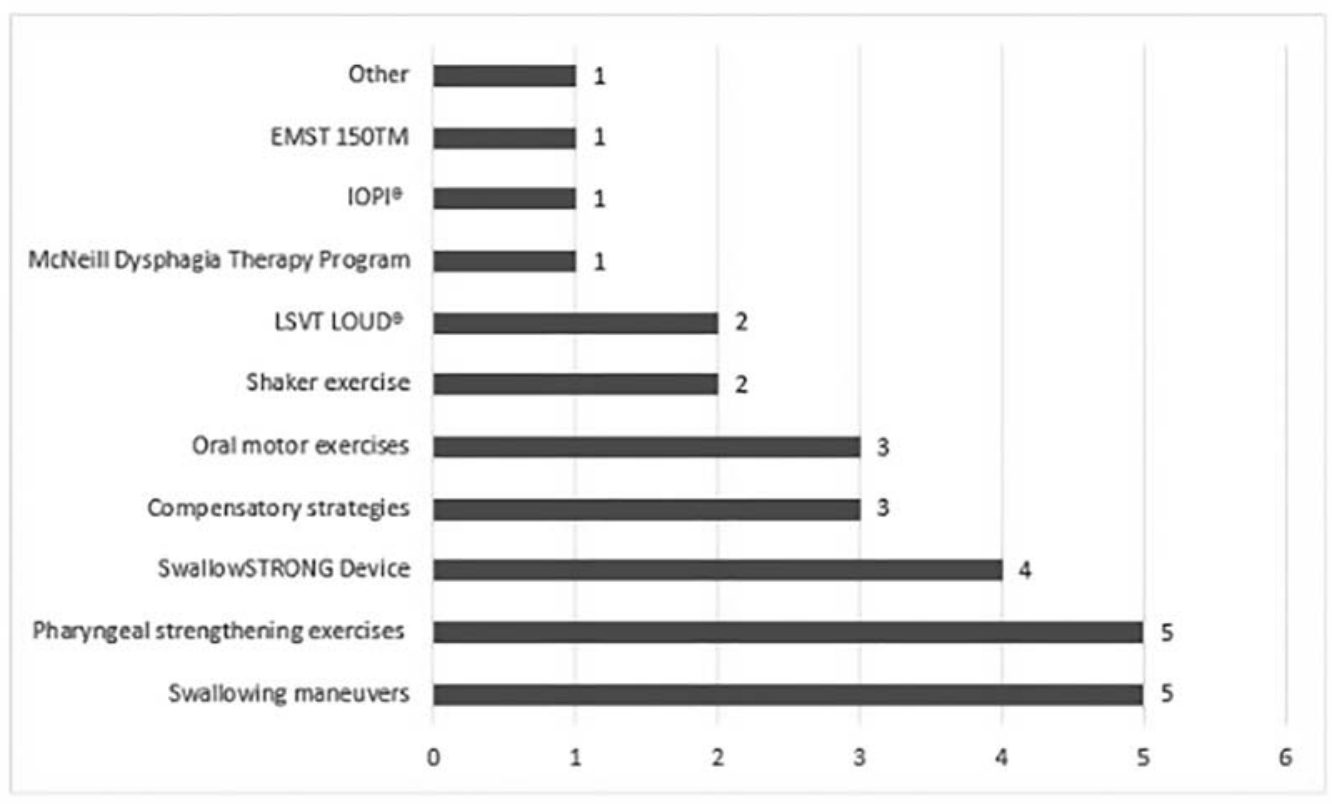

Figure 5 Most common dysphagia treatment approaches used with mental practice.

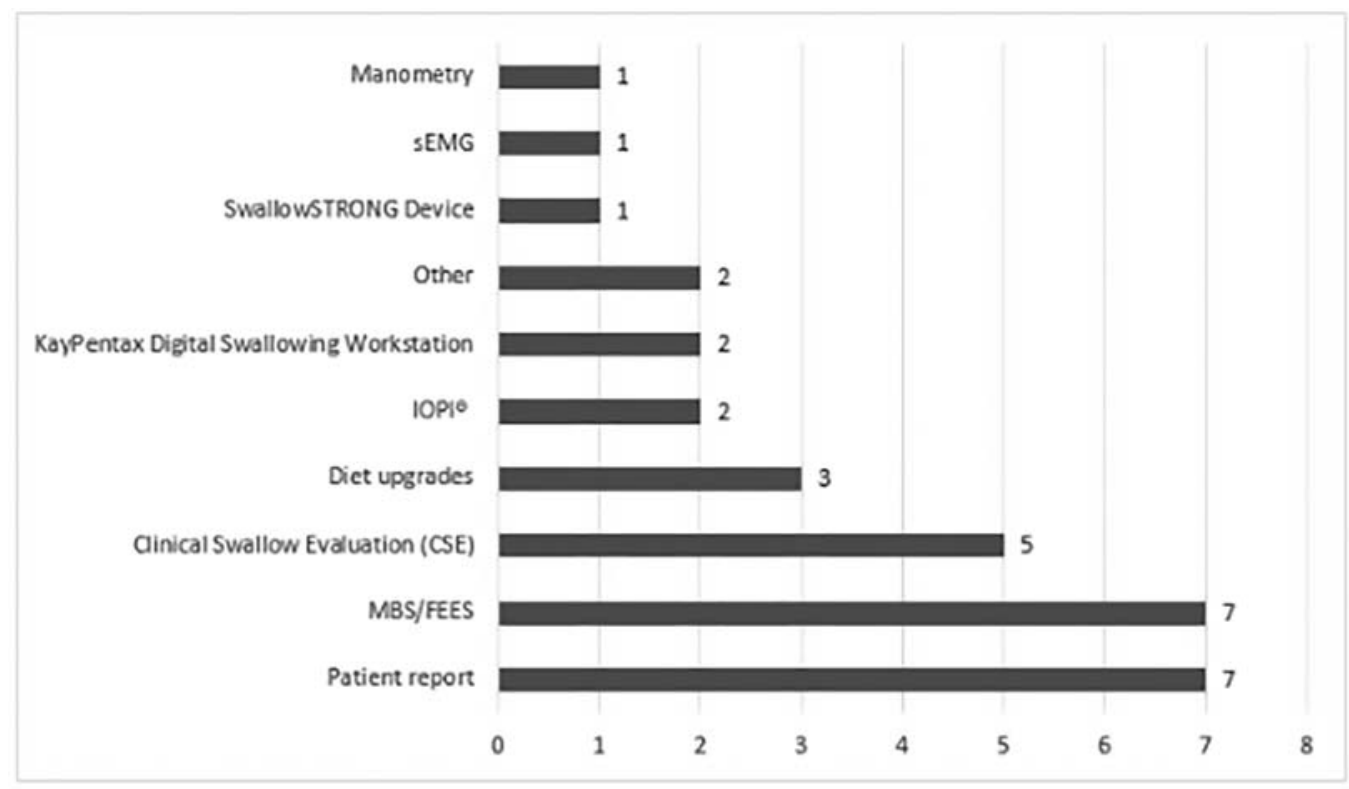

Figure 6 Methods used to measure patient progress and performance outcomes of mental practice.

Respondents reported that MP was advantageous to their patients to a very high degree $(n=1)$, high degree $(n=4)$, moderate degree $(n=4)$, and small degree $(n=2)$. They indicated that their patients responded to MP with a high degree $(n=1)$, moderate degree $(n=7)$, and small degree $(n=3)$. No respondents selected "Not at all" for either level of advantage or patient responsiveness. SLPs indicated several perceived advantages of using MP: safe for NPO (nil per os) patients $(n=6)$, ease of use for patient $(n=6)$, patient independence $(n=5)$, can be replicated or accessed at home $(n=5)$, patient confidence $(n=3)$, easy for SLP to demonstrate $(n=3)$, and patient may be familiar with technique from other disciplines and 


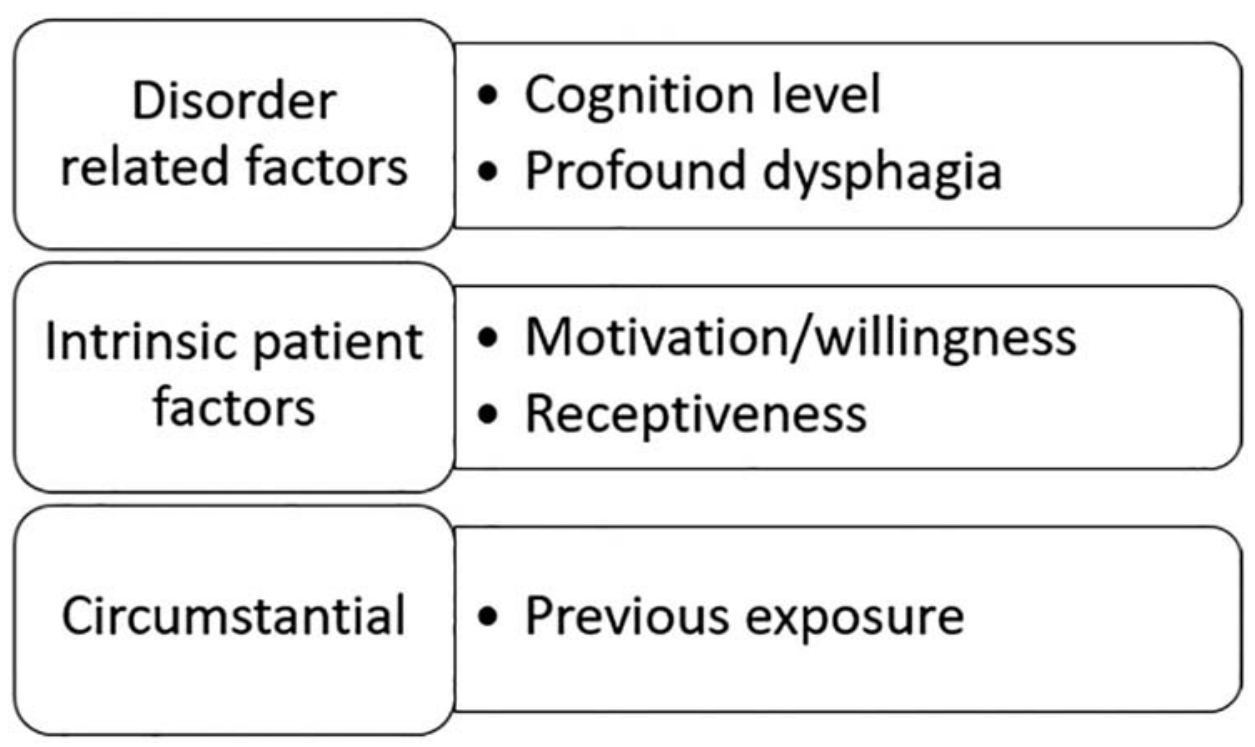

Figure 7 Potential themes for determining patient candidacy.

types of therapy $(n=2)$. One "Other" response was provided: increased practice time without fatigue. Perceived limitations of using MP were also identified: hard to monitor patient's mental technique $(n=9)$, requires sustained attention $(n=7)$, lack of patient understanding $(n=7)$, patient compliance $(n=5)$, not commonly used $(n=5)$, and not enough evidence-based research $(n=4)$. One "Other" response described concern of the SLP's own limited knowledge.

Respondents were asked via an open-ended question how they determined if a patient was a good candidate for MP. The 17 unique content units from the 10 respondents who completed this question were inductively coded. The greatest determiner was cognitive level of patient $(n=5)$, followed by ability to follow directions $(n=4)$, receptiveness/motivation of patient $(n=4)$, judgement of clinician $(n=3)$, and attendance $(n=1)$. The minimal number of codes and respondents limited organization into themes; however, three potential themes were noted: disorder-related factors, intrinsic patient factors, and circumstantial (see Fig. 7).

\section{Qualitative Analysis}

Interviews were not conducted; so, theme emergence was limited. However, the two openended questions analyzed earlier provide us with a deeper glimpse into patient candidacy and perceived challenges in using MP. The open-ended questions explored two unique questions (i.e., limitations for implementation and patient candidacy), but revealed overlapping emerging themes, as these topics are ultimately related. All the themes regarding patient candidacy (Fig. 7) are related to patient engagement, and therefore merge well with the largest theme that emerged from perceived limitations (Fig. 4). The themes have been revised slightly to more appropriately represent both patient candidacy considerations and perceived limitations jointly.

The first emerging theme involves factors that SLPs perceive to affect patient engagement. For example, responses show a primary concern for ability of the patient to mentally engage in MP. One respondent stated MP would be appropriate, "ideally if they have cognition and memory for independent practice between sessions." Another shared, "many of my clients have cognitive impairments, which would likely impact ability to do MP." While the presence of multiple handicaps was given as a perceived limitation, one SLP who uses MP shared that "profound dysphagia that makes traditional swallowing treatments more challenging" was a positive candidacy marker. Therefore, physical limitations, as well as cognitive, are perceived to affect patient engagement. Emphasis was placed on whether the patient was "receptive to the concept," "familiar with MP in other aspects of their lives," and "motivated." 
The second emerging theme involves evidence-based practice concerns, highlighting the lack of clinician knowledge and training, as well as the need for more research in this area. Respondents asked, "Is it research based? Will it improve outcomes?" They admitted they would "want to read the research first to assure [themselves] of the validity and efficacy of this approach." Subsequently, it would be important to "completely [understand] how to teach a patient how to complete [MP] successfully." One SLP who reported using MP admitted that they "did not even know that this was a therapy technique," which suggests that other SLPs may be using some concepts of MP without even knowing it. Finally, a third emerging theme of therapeutic environmental factors involved respondents noting factors such as insufficient therapy time and distracting therapeutic environments. One SLP expressed that he or she had "limited access to a low stim environment for working with residents," because MP may warrant a quiet environment until the patient acclimates to the technique.

\section{DISCUSSION}

General demographic data indicate that most survey participants were female, Caucasian, younger than 40 years, and working in multiple settings mainly with patients with a neurogenic etiology of dysphagia, with patients poststroke representing the largest category. The majority of SLP participants appear to use compensatory strategies and rehabilitative exercises with patients, with less focus on neuromuscular electrical stimulation and device-focused therapies. These data contribute to a better understanding of current dysphagia rehabilitation in general.

The most significant findings of the present survey study are the current interest in and familiarity with MP using motor imagery exercise. Nearly $75 \%$ of participants are interested in learning more about MP, over half are already at least somewhat familiar with the concepts of $\mathrm{MP}$, and nearly a quarter of the respondents are already incorporating MP into their clinical practice. These participants represent a group of underserved SLP clinicians-in other words, SLPs with the desire to use a clinical tool but lacking access to evidence-based research, train- ing, and education in the area of MP. Further investigation in this area would help address this need and provide evidence-based guidance to SLPs interested in incorporation of MP into management plans. Of those including MP already, none reported that MP was "not at all" useful for their patients; rather, all noted some level of advantage for their patients. This group also primarily used MP alongside traditional strengthening exercises. However, a low number responded to the question about how progress was monitored with patients and various dosing strategies were presented, further indicating the need for evidence-based guidance.

Close and open-ended responses to questions regarding perceived advantages, perceived limitations, patient candidacy, and factors affecting the likelihood to implement MP appeared to express repeated considerations important to our respondents. When responding to close-ended questions, respondents selected options that closely paired with those given in open-ended questions. Inadvertent leading of responses may have occurred due to survey item order. Open-ended questions were presented after closed-answer questions. However, the repeated emphasis on evidencebased practice, patient motivation, and patient's ability to participate provide a level of confidence in the data via triangulation.

In addition to the high level of interest in MP demonstrated by the survey respondents, these collective, perceived concerns point to the need for training about how to appropriately assess a patient's imaging ability (e.g., assessing cognitive ability, language ability, standardized imaging questionnaires, mental chronometry) and for a better understanding of which populations may benefit from inclusion of MP exercise in therapy. For example, imaging ability in the stroke population appears to improve within a few weeks postinjury, and the need to reassess imaging ability with this population is important. ${ }^{31}$ Patients with Parkinson's disease, a progressive neurological condition with associated deficits in control and motor learning, also appear to have well-preserved motor imaging ability prior to the late disease stage. ${ }^{32}$ The current investigators are unsure about specific sources for current knowledge of MP claimed by a 
large number of respondents, though interaction with other disciplines appears to be a potential source. However, the high level of interest in the present preliminary data and emerging evidence involving the use of MP in the area of dysphagia signals the need for increased support, evidence, and training opportunities for SLPs specifically interested in implementing MP exercise concepts in intervention management plans. $^{22,24-28,30}$

\section{LIMITATIONS}

These data are best viewed as preliminary findings given the small sample size of survey respondents. The sample of participants in the present survey study represents the different geographic regions of the United States fairly well; however, more information is needed from the Pacific, Mountain West, Midwest, and South Atlantic regions of the United States as well as data from different countries, to better understand any relationship between geographical location and knowledge/practice patterns of MP. The survey did not allow for a deeper exploration via interviews, as broad survey reach across the United States was important for this initial exploration. The present study's sample, which represented a cross-section of individuals drawn from across the United States, provided a preliminary understanding of MP in the area of dysphagia. However, specific group comparisons were not made as many questions were not designed for more specific comparison using statistical analysis and respondent groups were not distributed well for meaningful comparison.

Regarding the implementation and knowledge of MP, two limitations are noted. First, even though we provided a frame of reference regarding MP using motor imagery at the start of the survey, it is probable that respondents have varying ideas about this concept as it is new to the field of SLP. Second, the benefits of MP from participants were subjectively reported, without further explanation of how such benefits were measured. While practitioner expertise and patient preferences are part of evidencebased practice, the component of research evidence (well-designed efficacy studies) is necessary to comprehensively support MP in clinical practice.

\section{SUMMARY}

Development of innovative approaches, such as $\mathrm{MP}$, to enhance and improve current rehabilitative methods in dysphagia is needed. ${ }^{24,33}$ This study presents preliminary data to highlight the interest of SLPs in learning more about MP and the needs for increased resources to support these clinicians and their patients. Future directions should include research specific to swallowing-related musculature and tasks, with potential reach of implementation of MP beyond application to only strengthening exercises. Several of the perceived advantages of using MP were captured by the current group of respondents, that is, safe for NPO patients, ease of use, patient independence, access in the home environment, and patient confidence. MP may potentially benefit different dysphagic populations (e.g., poststroke, head/neck cancer, ALS, Parkinson's disease) and tasks beyond enhancement of strengthening exercises (e.g., MP as a priming exercise or simulated practice environment for swallowing and swallowingrelated tasks for patients who are at a high risk of aspiration with any oral intake, priming exercise prior to instrumental evaluation of swallowing function or oral intake).

\section{CONFLICT OF INTEREST}

None declared.

\section{REFERENCES}

1. Namasivayam-MacDonald AM, Shune SE. The burden of dysphagia on family caregivers of the elderly: a systematic review. Geriatrics (Basel) 2018;3(02):30

2. Shune SE, Namasivayam-MacDonald AM. Swallowing impairments increase emotional burden in spousal caregivers of older adults. J Appl Gerontol 2020;39(02):172-180

3. Logemann JA. Swallowing disorders. Best Pract Res Clin Gastroenterol 2007;21(04):563-573

4. O'Keeffe ST. Use of modified diets to prevent aspiration in oropharyngeal dysphagia: is current practice justified? BMC Geriatr 2018;18(01):167

5. Langmore SE, Pisegna JM. Efficacy of exercises to rehabilitate dysphagia: a critique of the literature. Int J Speech Lang Pathol 2015;17(03):222-229

6. McKenna VS, Zhang B, Haines MB, Kelchner LN. A systematic review of isometric lingual strength-training programs in adults with and 
without dysphagia. Am J Speech Lang Pathol 2017;26(02):524-539

7. Rogus-Pulia N, Churness K, Hind J, Gangnon R, Banaszynski K, Robbins J. Comparison of maximal lingual pressure generation during isometric gross and fine sensorimotor tasks in healthy adults. Arch Phys Med Rehabil 2015;96(10):1785-1794

8. Collet C, Guillot A. The Neurophysiological Foundations of Mental and Motor Imagery. Oxford: Oxford University Press; 2010

9. Decety J. The neurophysiological basis of motor imagery. Behav Brain Res 1996;77(1-2):45-52

10. Jeannerod M. Mental imagery in the motor context. Neuropsychologia 1995;33(11):1419-1432

11. Jeannerod $M$. The representing brain: neural correlates of motor intention and imagery. Behav Brain Sci 1994;17(02):187-202

12. Decety J, Ingvar DH. Brain structures participating in mental simulation of motor behavior: a neuropsychological interpretation. Acta Psychol (Amst) 1990;73(01):13-34

13. Driskell JE, Copper C, Moran A. Does mental practice enhance performance? J Appl Psychol 1994;79:481-492

14. Moran A, Guillot A, Macintyre T, Collet C. Reimagining motor imagery: building bridges between cognitive neuroscience and sport psychology. $\mathrm{Br} \mathrm{J}$ Psychol 2012;103(02):224-247

15. Richardson A. Mental practice: a review and discussion, part 1. Res Q 1967;38:95-107

16. Dickstein R, Deutsch JE. Motor imagery in physical therapist practice. Phys Ther 2007;87(07):942-953

17. Peters HT, Page SJ. Integrating mental practice with task-specific training and behavioral supports in poststroke rehabilitation: evidence, components, and augmentative opportunities. Phys Med Rehabil Clin N Am 2015;26(04):715-727

18. Slimani M, Tod D, Chaabene H, Miarka B, Chamari K. Mental imagery on muscular strength in healthy and patient participants: a systematic review. J Sports Sci Med 2016;15(03):434-450

19. Tod D, Edwards C, McGuigan M, Lovell G. A systematic review of the effect of cognitive strategies on strength performance. Sports Med 2015;45 (11):1589-1602

20. Ruffino C, Papaxanthis C, Lebon F. Neural plasticity during motor learning with motor imagery practice: review and perspectives. Neuroscience 2017;341:61-78

21. Page SJ, Harnish S. Thinking about better speech: mental practice for stroke-induced motor speech impairments. Aphasiology 2012;26(02):127-142
22. Szynkiewicz SH, Nobriga CV, O'Donoghue CR, Becerra BJ, LaForge G. Motor imagery practice and increased tongue strength: a case series feasibility report. J Speech Lang Hear Res 2019;62(06): 1676-1684

23. Ehrsson HH, Geyer S, Naito E. Imagery of voluntary movement of fingers, toes, and tongue activates corresponding body-part-specific motor representations. J Neurophysiol 2003;90(05): 3304-3316

24. Jestrović I, Coyle JL, Sejdić E. Decoding human swallowing via electroencephalography: a state-ofthe-art review. J Neural Eng 2015;12(05):051001

25. Kober SE, Grössinger D, Wood G. Effects of motor imagery and visual neurofeedback on activation in the swallowing network: a real-time fMRI study. Dysphagia 2019;34(06):879-895

26. Kober SE, Bauernfeind G, Woller C, et al. Hemodynamic signal changes accompanying execution and imagery of swallowing in patients with dysphagia: a multiple single-case near-infrared spectroscopy study. Front Neurol 2015;6:151

27. Kober SE, Gressenberger B, Kurzmann J, Neuper C, Wood G. Voluntary modulation of hemodynamic responses in swallowing related motor areas: a near-infrared spectroscopy-based neurofeedback study. PLoS One 2015;10(11):e0143314

28. Kober SE, Wood G. Changes in hemodynamic signals accompanying motor imagery and motor execution of swallowing: a near-infrared spectroscopy study. Neuroimage 2014;93(Pt 1):1-10

29. Stippich C, Ochmann H, Sartor K. Somatotopic mapping of the human primary sensorimotor cortex during motor imagery and motor execution by functional magnetic resonance imaging. Neurosci Lett 2002;331(01):50-54

30. Yang H, Ang KK, Wang C, Phua KS, Guan C. Neural and cortical analysis of swallowing and detection of motor imagery of swallow for dysphagia rehabilitation - a review. Prog Brain Res 2016; 228:185-219

31. de Vries S, Tepper M, Otten B, Mulder T. Recovery of motor imagery ability in stroke patients. Rehabil Res Pract 2011;2011:283840

32. Heremans E, Feys P, Nieuwboer A, et al. Motor imagery ability in patients with early- and midstage Parkinson disease. Neurorehabil Neural Repair 2011;25(02):168-177

33. Jadcherla SR, McCullough GH, Daniels SK, et al. The Dysphagia Research Society accelerating a priority research agenda. Dysphagia 2017;32(01): 11-14 


\section{APPENDIX A}

Please read the information below and answer the following questions based on what you have read.

Motor imagery is the act of imagining oneself or another performing a motor task without actually performing physical action. Mental practice is the repetitive implementation of a motor imagery task. Mental practice using motor imagery serves as a cognitive rehabilitation tool to enhance physical practice. Mental practice is used with neurogenic patient populations (e.g., stroke, Parkinson's disease) in the fields of physical and occupational therapy to improve motor performance in areas such as strength, posture, balance, and activities of daily and independent living.

Before incorporating mental practice to the therapy plan, the therapist educates the patient about its use. Then, the patient and therapist work together to determine if they are a good imager and will benefit from mental practice. If the patient meets both criteria, he or she is typically instructed to complete mental practice using motor imagery by (1) alternating physical and mental practice repetitions, (2) completing mental practice directly following active practice, or (3) completing mental practice later in the day in the home setting.

Mental practice has been used to visualize and practice skills in many different areas such as:

- Reaching for a cup.

- Playing a musical instrument.

- Preparing for an athletic competition.

- Surgical training.

- Strengthening a muscle of the limbs.

Using mental practice and motor imagery in the field of speech-language pathology (SLP) is starting to be explored. Specifically, in the area of dysphagia, research is being conducted to determine if mental practice can help increase tongue strength. For the remainder of the survey, we will use the term "mental practice" instead of "mental practice with motor imagery." We want to learn more about what SLPs know about mental practice, if/how it is currently used in practice, and learn about how you think mental practice could be beneficial to patients.

1. Are you familiar with mental practice?
A. Yes.
B. No.
C. Somewhat.

2. Do you know of other SLPs who use mental practice?
A. Yes.
B. No.
C. Not sure.

3. Do you know of professionals from other disciplines (e.g., PT/OT) who use mental practice?
A. Yes.
B. No.
C. Not sure.

4. Do you think your patients would be willing to incorporate mental practice with their conventional dysphagia rehabilitative exercises?
A. Yes.
B. No.
C. Not sure.

5. What are some challenges (if any) that would deter you from implementing mental practice? 
6. What are some of the most common technique you use to treat dysphagia? Select all that apply.

\begin{tabular}{|c|c|}
\hline a. NMES (VitalStim) & b. Oral motor exercises \\
\hline c. Shaker exercise & d. Pharyngeal strengthening exercises \\
\hline e. McNeill Dysphagia Tx Program & f. IOPI \\
\hline g. LSVT LOUD & h. SwallowSTRONG Device \\
\hline i. Swallowing maneuvers & j. sEMG \\
\hline k. Compensatory strategies & I. EMST 150 \\
\hline m. Diet modification & n. Other: \\
\hline o. Thermal/tactile stimulation & \\
\hline
\end{tabular}

7. Have you used mental practice with a patient before?

A. Yes (if yes, they were not presented questions 12-21).

B. No (if no, they were not presented questions 8-11).

8. How applicable do you think this treatment approach would be for your patient population?
A. Very high degree.
B. High degree.
C. Moderate degree.
D. Small degree.
E. Not at all.

9. To what extent could mental practice supplement your existing therapy technique?
A. Very high degree.
B. High degree.
C. Moderate degree.
D. Small degree.
E. Not at all.

10. How likely are your patients to respond to the idea of mental practice?
A. Very high degree.
B. High degree.
C. Moderate degree.
D. Small degree.
E. Not at all.

11. What are some factors that may affect your likelihood to implement or try mental practice with motor imagery? Select all that apply.
A. Evidence in literature.
B. Perception by others in your field.
C. Ease of implementation.
D. Self-efficacy for patient.
E. Other:

12. External imagery is when a person views him/herself from the perspective of an external observer and internal imagery is when a person imagines being inside his/her body and experiencing those sensations that might be expected in the actual situation. When you train mental practice, do you differentiate between external or internal motor imagery?
A. Yes.
B. No.
C. Not sure. 
13. What kind of therapy tasks do you use mental practice approaches with for your patients? Select all that apply.

a. Shaker exercise

b. McNeill Dysphagia Tx Program

c. LSVT LOUD

d. Swallowing maneuvers

e. Compensatory strategies

f. Oral motor exercises

g. Pharyngeal strengthening exercises

h. IOPI

i. SwallowSTRONG Device

j. EMST 150

k. Other:

14. How do you determine if a patient is a good candidate for mental practice?

15. How do you incorporate mental practice techniques into your patient's therapeutic plan? Select all that apply.
A. Alternate between mental and physical exercise repetitions or sets within a session.
B. Session is divided into two parts: mental and physical exercise.
C. Alternate days of mental and physical exercise.
D. Mental exercise is only completed outside of therapy sessions.
E. Other:

16. What is the typical schedule for patients using mental practice?
A. Several time per day.
B. Twice per day.
C. Daily.
D. More than 5 times per week.
E. 3-5 times per week.
F. Less than 3 times per week.

17. How do you monitor patient progress and performance outcome of mental practice with motor imagery? Select all that apply.
a. IOPI
b. Patient report
c. Diet upgrades
d. MBS/FEES
e. Clinical swallow evaluation (CSE)
f. SwallowSTRONG Device
g. Semg
h. Manometry
i. KayPentax Digital Swallowing Workstation
j. Other:

18. How advantageous do you perceive mental practice to be with your patients?
A. Very high degree.
B. High degree.
C. Moderate degree.
D. Small degree.
E. Not at all.

19. How do your patients respond to mental practice?
A. Very high degree.
B. High degree.
C. Moderate degree.
D. Small degree.
E. Not at all. 
20. What are some of the advantages of using mental practice with motor imagery? Select all that apply.

a. Patient independence

c. Can be replicated/accessed at home

e. Safe for NPO patients

g. Patient may be familiar with approach from PT/OT b. Ease of use for patient

d. Easy for SLP to demonstrate

f. Patient confidence

h. Other:

21. What are some of the limitations of using mental practice? Select all that apply.

The remaining questions were demographics of all participants. Question content can be determined from the "Results" section of this article.
a. Not enough evidence-based research
b. Not commonly used
c. Patient compliance
d. Lack of patient understanding
e. Hard to monitor patient's mental technique
f. Requires sustained attention
g. Other: 